\title{
Ablation plasma ion implantation experiments: Measurement of Fe implantation into $\mathrm{Si}$
}

\author{
B. Qi, R. M. Gilgenbach, ${ }^{\text {a) }}$ Y. Y. Lau, M. D. Johnston, J. Lian, and L. M. Wang \\ Intense Energy Beam Interaction Laboratory, Nuclear Engineering and Radiological Sciences Department, \\ University of Michigan, Ann Arbor, Michigan 48109-2104
}

G. L. Doll and A. Lazarides

Advanced Materials $R \&$ D, Timken Research, The Timken Corporation, Canton, Ohio 44706-0930

(Received 2 March 2001; accepted for publication 24 April 2001)

\begin{abstract}
Experiments have been performed demonstrating the feasibility of direct implantation of laser-ablated metal ions into a substrate. Initial experiments implanted iron ions into silicon substrates at pulsed, bias voltages up to negative $10 \mathrm{kV}$. Implantation of Fe ions into $\mathrm{Si}$ was confirmed by cross-sectional transmission electron microscopy and $\mathrm{X}$-ray photoelectron spectroscopy. The $7.6 \mathrm{~nm}$ depth of damage layers below the Si surface is slightly less than predicted by code calculations for a maximum, effective ion energy of about $8 \mathrm{keV}$. The ion depth of penetration is limited by the overlying Fe film as well as the slow rise and fall of the voltage. (C) 2001 American Institute of Physics. [DOI: 10.1063/1.1379360]
\end{abstract}

Over the past decade, plasma-immersed-ion-implantation (PIII) has become an established materials processing technique. $^{1-10}$ In conventional PIII, a gaseous precursor (most typically nitrogen) is ionized to serve as the source of ions for implantation. Pulsed (ablative) laser deposition (PLD) is also an accepted technique for deposition of thin films, ${ }^{11}$ in some cases using a bias voltage to alter the properties of (e.g., diamond-like carbon) films. ${ }^{12}$ In ablation plasma ion implantation (APII), ${ }^{13,14}$ a solid target is ablated by a laser; the resulting plasma plume is the source of ions, which are accelerated to high energy by the pulsed, negatively biased substrate. APII performs ion beam assisted deposition of thin films. Thus, APII has several advantages, the most important being direct metal ion implantation with no toxic gaseous precursors. Recently, theories ${ }^{14,15}$ were elucidated for APII. Scaling laws have been constructed for the APII maximum achievable dose and the ion current as a function of the plume-substrate spacing. ${ }^{14}$ In this letter, we demonstrate the feasibility of ion implantation utilizing laser ablated metal plasmas as the ion source.

The experimental configuration is depicted in Fig. 1. A KrF laser (Lambda Physik Compex 205) generates up to 600 $\mathrm{mJ}$ in a $20 \mathrm{~ns}$ pulse at a wavelength of $248 \mathrm{~nm}$. The laser is focused onto a pure-iron target, which is pyramid-shaped and rotating $(15 \mathrm{rpm})$ in order to wobble the ablation plume for deposition over a larger area on the silicon substrate. The target-to-substrate normal spacing is set at $3.7 \mathrm{~cm}$. The negative output pulse from a high voltage generator is fed into the vacuum chamber through a $100 \mathrm{kV}$ rated insulator; the $\mathrm{Si}$ (100) substrate is mounted on the end of a rod passing through this insulator. The delay between the laser pulse and the high voltage pulse is controlled by a digital delay generator; a repetition rate of $1-10 \mathrm{~Hz}$ is employed. The experiment is evacuated by a turbomolecular pump to the $10^{-6}$ Torr scale. A high voltage probe and current trans-

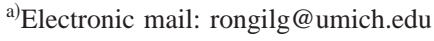

former (Pearson) are utilized as electrical diagnostics. Optical emission spectroscopy is performed by a $0.3 \mathrm{~m}$ spectrograph with gated, intensified charge coupled device detector; atomic Boltzmann plots for the laser ablated $\mathrm{Fe}$ plasma have yielded a peak electronic temperature of $12 \mathrm{eV}$ cooling to the eV level on a microsecond time scale. ${ }^{16}$

Typical electrical signals are presented in Fig. 2. The PIN diode [sharp spike in Fig. 2(a)] monitors the timing of the ablation laser pulse. The voltage pulse (without plasma) is displayed in Fig. 2(c), showing the peak applied voltage up to negative $10 \mathrm{kV}$ for typically $10 \mu$ s total pulse duration. The voltage pulse with laser ablation plasma is displayed in Fig. 2(d); these data show the plasma loading effect on the power supply, which lowers the peak voltage and decreases the RC decay time of the voltage. For these data, the relative delay between the laser and the voltage pulse [Figs. 2(a)$2(d)]$ is set at about $7 \mu$ s. This delay was necessary to prevent arcing between the substrate and target; such arcing has been shown to punch holes in the deposited film. The current

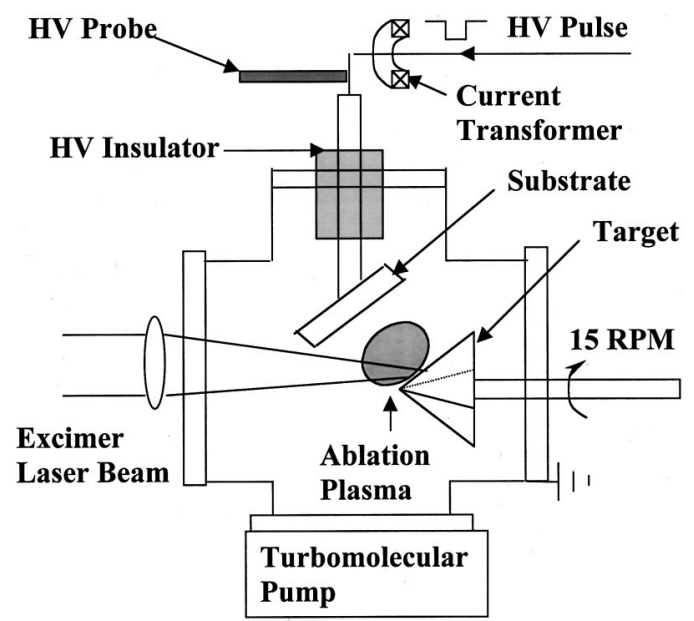

FIG. 1. APII experimental configuration. The spacing between the target and substrate is $3.7 \mathrm{~cm}$. 


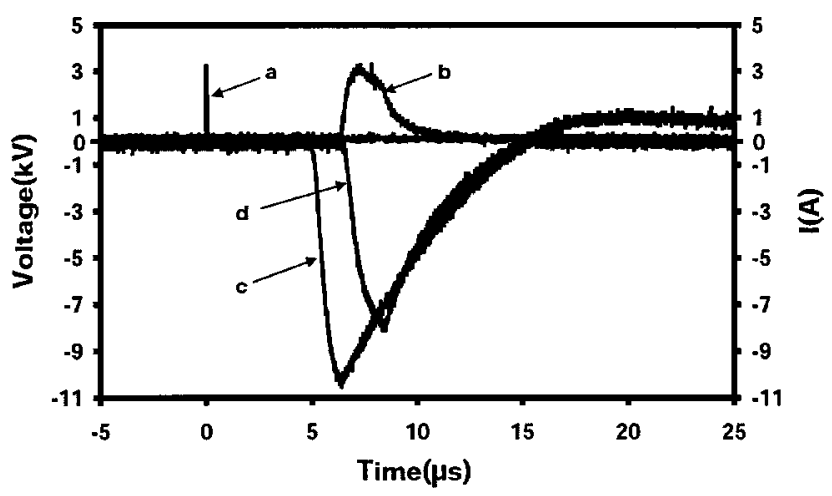

FIG. 2. Electrical diagnostic signals: (a) pin diode (laser pulse timing), (b) current transformer (net positive current) with displacement current (no plasma) subtracted, (c) high voltage pulse without plasma, and (d) high voltage pulse with plasma.

transformer signal [Fig. 2(b)] represents the sum of the electron current (accelerated away from the substrate) and the implanted ion current. The displacement current (without laser) has been digitally subtracted here, so this signal is zero in the absence of ablation plasma.

Experiments were performed in two series. First, baseline samples consisted of laser ablated iron deposited onto silicon substrates with zero applied voltage. Second, APII films were generated by laser ablation plumes followed by high voltage pulses. The number of pulses, typically 2000 here, (up to 24000 for other studies), depended upon the desired thickness of the deposited film. Deposited films were analyzed by cross-sectional transmission electron microscopy (TEM) and x-ray photoelectron spectroscopy. Additional tests were performed of film adhesion, which will not be presented here.

A cross-sectional TEM photograph of a baseline film is shown in Fig. 3, as deposited by 2000 laser pulses with zero voltage. These TEM data show that the deposited film consists of an iron silicide transition layer (b) on the crystalline silicon substrate (a), with an overlying region (c) containing nanocrystalline Fe. Such iron silicide layers have been deposited in previous PLD experiments. ${ }^{11}$ For zero accelerating

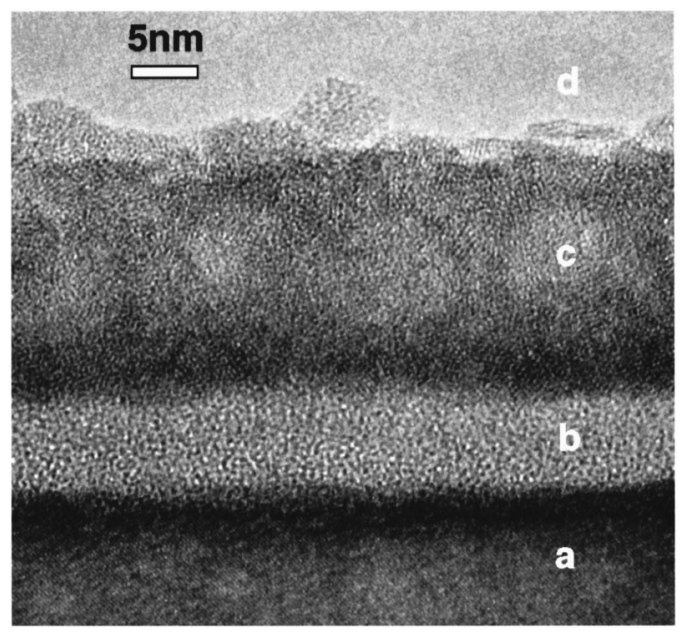

FIG. 3. Cross-sectional TEM photograph of baseline (zero voltage) laser deposited film. (a) Single crystal Si substrate, (b) amorphous iron silicide transition layer, (c) nanocrystalline Fe layer, and (d) glue.

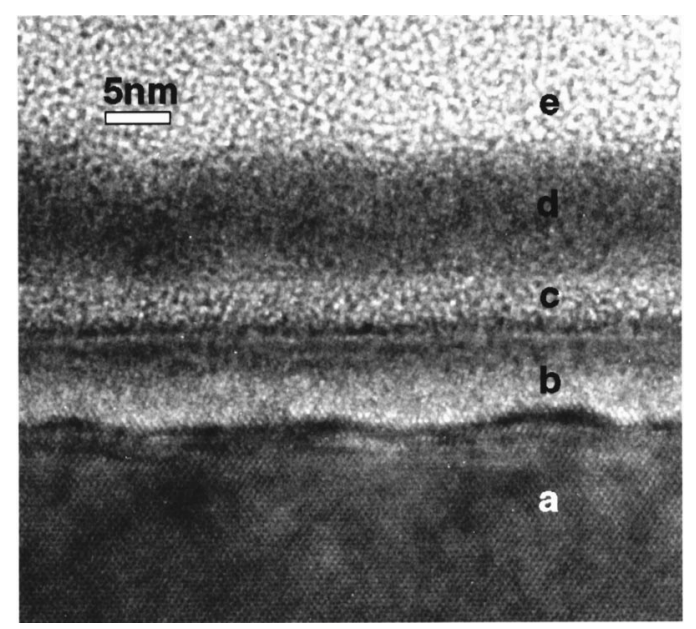

FIG. 4. Cross-sectional TEM photograph of APII ( $-10 \mathrm{kV}$ voltage) deposited film. (a) Single crystal Si substrate, (b) damage layer, (c) amorphous iron silicide transition layer, (d) amorphous Fe layer, and (e) glue.

voltage with low energy (electron volt) laser plasma ions there is no damage layer below the surface of the Si.

Figure 4 presents cross-sectional TEM data for ablation plasma iron ions implanted into silicon with 2000 pulses at a peak applied voltage of $-10 \mathrm{kV}$ for a total pulse duration of $10 \mu \mathrm{s}$. The most important difference from the baseline film is that APII sample shows a damage layer (b) that extends some $7.6 \mathrm{~nm}$ below the original surface of the silicon [(b)(c) interface]. The damaged layer is believed to be the result of collisional cascades. ${ }^{17}$ The film (c) directly above the silicon surface is amorphous iron silicide. The top iron layer (d) of the APII deposited film is also amorphous, due to ion bombardment. The experimentally observed $7.6 \mathrm{~nm}$ penetration depth is slightly less than that predicted by the SRIM (stopping and range of ions in matter) $\operatorname{code}^{18}$ for a peak ion energy of about $8 \mathrm{kV}$. Thus, the shallower experimental ion penetration depth is caused by two factors:

(1) all but the initial, accelerated Fe ion pulses must pass through the overlying deposited Fe film, and

(2) the applied voltage consists of a slow rise and fall time. ${ }^{19}$

Figure 5 depicts APII film composition data obtained from x-ray photoelectron spectroscopy. These data are presented as a function of time while performing Ar ion sputtering of the film, so the data provide a depth profile of species. It is apparent that at zero sputtering time (at the surface) there exists primarily Fe, with low concentrations of

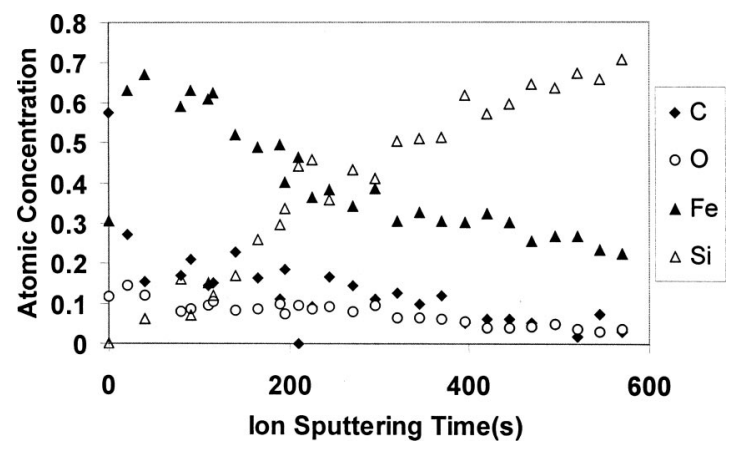

FIG. 5. Atomic concentration vs Ar ion sputtering time (film depth) obtained from x-ray photoelectron spectroscopy. 
$\mathrm{Si}$ (along with $\mathrm{C}$ and $\mathrm{O}$ impurities). As the sputtering time (depth) increases the film shows a gradual change from $\mathrm{Fe}$ to iron silicide, with roughly equal concentrations of $\mathrm{Fe}$ vs $\mathrm{Si}$ between 200 and $300 \mathrm{~s}$. At later times (depths), the concentration makes a transition to mainly $\mathrm{Si}$ with lower concentrations of implanted $\mathrm{Fe}$. These data clearly demonstrate iron implantation into bulk silicon, verifying the feasibility of APII as an ion acceleration and implantation process.

The authors appreciate funding by The National Science Foundation, Grant No. CTS-9907106. The authors also thank S. M. Yalisove for expert advice.

${ }^{1}$ J. R. Conrad, J. L Radtke, R. A. Dodd, F. J. Worzala, and N. C. Tran, J. Appl. Phys. 62, 4591 (1987).

${ }^{2}$ J. T. Sheuer, M. Shamim, and J. R. Conrad, J. Appl. Phys. 67, 1241 (1990).

${ }^{3}$ B. P. Wood, I. Henins, D. J. Rej, H. A. Davis, W. Waganaar, R. E. Muenchausen, G. P. Johnson, and H. K. Schmidt, Nucl. Instrum. Methods Phys. Res. B 96, 429 (1995).

${ }^{4}$ P. K. Chu, S. Qin, C. Chan, N. W. Cheung, and L. A. Larson, Mater. Sci. Eng., R. 17, 207 (1996).

${ }^{5}$ M. A. Lieberman, J. Appl. Phys. 66, 2926 (1989).
${ }^{6}$ M. A. Lieberman and A. J. Lichtenberg, Principles of Plasma Discharges and Material Processing (Wiley, New York, 1994), Chap. 6.4.

${ }^{7}$ B. P. Cluggish and C. P. Munson, J. Appl. Phys. 84, 5937 (1998).

${ }^{8}$ B. P. Cluggish and C. P. Munson, J. Appl. Phys. 84, 5956 (1998).

${ }^{9}$ E. C. Jones and N. W. Cheung, IEEE Trans. Plasma Sci. 25, 42 (1997).

${ }^{10}$ Handbook of Plasma Immersion Ion Implantation edited by A. Anders, (Wiley, New York, 2000).

${ }^{11}$ O. P. Karpenko, C. H. Olk, S. M. Yalisove, J. F. Mansfield, and G. L. Doll, J. Appl. Phys. 76, 2202 (1994).

${ }^{12}$ N. Matsuyama, K. Yukimura, and T. Maruyama, J. Appl. Phys. 89, 1938 (2001).

${ }^{13}$ R. M. Gilgenbach, B. Qi, Y. Y. Lau, and M. D. Johnston, Conference Record of the 2000 IEEE International Conference on Plasma Science, June 4-7, 2000, New Orleans, LA, IEEE Catalog Number 00CH37087, p. 209.

${ }^{14}$ B. Qi, Y. Y. Lau, and R. M. Gilgenbach, Appl. Phys. Lett. 78, 706 (2001).

${ }^{15} \mathrm{~S}$. Masamune and K. Yukimura, Rev. Sci. Instrum. 71, 1187 (2000).

${ }^{16}$ R. M. Gilgenbach, B. Qi, Y. Y. Lau, and M. D. Johnston, Bull. Am. Phys. Soc. 45, 230 (2000)

${ }^{17}$ Ion Solid Interactions: Fundamentals and Applications, edited by M. Nastasi, J. W. Mayer, and J. K. Hirvonen, (Cambridge University Press, Cambridge, 1996), p. 147.

${ }^{18}$ J. F. Ziegler and J. P. Biersack. SRIM-2000.39 Version available at http:// www.research.ibm.com/ionbeams.

${ }^{19}$ R. A. Stewart and M. A. Lieberman, J. Appl. Phys. 70, 3481 (1991). 